\title{
Is endomyocardial biopsy necessary in evaluating enlarged hearts in athletes?
}

\author{
S.E. Trotter and E.G.J. Olsen
}

\section{Department of Cardiac Pathology, National Heart and Chest Hospitals, Brompton Hospital, Fulham Road, London SW3 6HP, UK}

The clinical manifestations of the 'athletic heart' have been well documented and a systolic murmur, bradycardia and cardiomegaly are observable features $;{ }^{1-4}$ evidence has shown that morphological adaptations occur in these hearts and it has also been recorded that these adaptive mechanisms depend on the type of exercise undertaken. ${ }^{4-8}$ Athletes engaged in static muscle contraction pursuits such as weight lifting have an increase in left ventricular thickness without dilatation of the cavities. ${ }^{3-5,7,8}$ Those individuals undertaking swimming or running have a dilated left ventricular cavity in the absence of any increase in wall thickness. ${ }^{4-8}$ To these two groups the terms isometric and isotonic exercise have been proposed. ${ }^{4}$ It can therefore be concluded that the isometric participators have a pressure load on the heart, whilst the isotonic group have a volume burden. ${ }^{4}$ In practice, most exercise involves a mixture of these two forms but it is considered that weight lifting represents predominantly isometric activity and swimming predominantly isotonic exercise. ${ }^{4,8}$

As well as a cardiomegaly, systolic murmur, and bradycardia, ${ }^{1-4}$ electrocardiographic abnormalities have also been described with the athletic heart. ${ }^{2}$ These clinical features, however, may be an expression of serious underlying pathology and differentiation is highly important, especially as sudden death in sports participators is well documented. ${ }^{4,9}$ Invasive investigations such as cardiac catheterization, may be necessary ${ }^{2}$ but the use of the bioptome to obtain fresh endomyocardial tissue, allowing morphological interpretation, may also permit differentiation of the physiological from the pathological. In isometric participators the main problem of exclusion is hypertrophic cardiomyopathy. It has been documented that in the hearts of sufferers with hypertrophic cardiomyopathy, the ratio of the thickness of the septal to posterior walls often exceeds $1.3 .{ }^{10}$ Similar findings are also seen in the hearts of isometric par-

Correspondence: S.E. Trotter, M.B., B.S.

Received: 10 May 1990 ticipators ${ }^{3,8}$ and this, together with the increase in ejection fraction and a small ventricular cavity seen in these athletes, ${ }^{8}$ makes differentiation from hypertrophic cardiomyopathy mandatory. Recourse to endomyocardial biopsy may be useful in these circumstances. In physiological hypertrophy there is an increase in diameter, indicating hypertrophy, but normal alignment of the myocardial fibres; attenuation, indicating dilatation of the myocardium; is not identifiable. By contrast, hypertrophic cardiomyopathy is characterized by disarray of the myofibres, extreme hypertrophy and nuclear changes, often surrounded by a clear zone. " Although some overlap between these changes and those of ordinary hypertrophy existso the histological HOCM index, formulated many years ago, allows differentiation and accurate diag nosis. ${ }^{12}$

It is of interest that Maron and his colleagues who examined the causes of sudden death in 29 highly trained athletes aged between 13 and 30 years, found structural abnormalities of the cardiovascular system in all but one of the competitors. ${ }^{9}$ The most common cause was hypertrophic cardiomyopathy, identified in 14 of the athletes. Other causes of pathology in athletes have been reported by Virmani and Robinowitz ${ }^{4}$ who examined 114 previously documented cases of joggers and marathon runners who died suddenly, and found coronary atherosclerosis as the cause in 83 of these.

In isotonic exercise a different picture exists; the cardiomegaly in these subjects is characterized by dilatation of the cavities, ${ }^{4-8}$ which is also a feature of dilated cardiomyopathy, which may be asymptomatic and discovered on routine examination. A careful history of the extent and severity together with non-invasive investigations may differentiate, but endomyocardial biopsy may be necessary. Unfortunately, the morphological changes in dilated cardiomyopathy are non-specific and delineation is impossible, unless additional features are present, such as myocarditis, which is now known to contribute to this form of cardiomyopathy. ${ }^{13}$ Follow up in these cases is essential.

It is evident that exercise can result in clinical 
manifestations, as well as adaptive changes. Accordingly it is the clinicians' responsibility to be aware of these mechanisms. Wrong interpretation of these clinical observations may result in the patient curtailing his or her sporting pursuits and furthermore, forfeit of occupation. Nevertheless, in some instances pathology may be the underlying cause and may be asymptomatic or elaborating

\section{References}

1. Gott, P.H., Roselle, H.A. \& Crampton, R.S. The athletic heart syndrome. Arch Intern Med 1968, 122: 340-344.

2. Oakley, M.A. \& Oakley, C.M. Significance of abnormal electrocardiograms in highly trained athletes. Am J Cardiol 1982, 50: 985-989.

3. Roeske, W.R., O'Rourke, R.A., Klein, H., Leopold, G. \& Karliner, J.S. Noninvasive evaluation of ventricular hypertrophy in professional athletes. Circulation 1976, 53: 286-292.

4. Virmani, R. \& Robinowitz, M. Cardiac pathology and sports medicine. Hum Pathol 1987, 18: 493-501.

5. Crawford, M.H., Petru, M.A. \& Rabinowitz, C. Effect of isotonic exercise training on left ventricular volume during upright exercise. Circulation 1985, 72: 1237-1243.

6. Colan, S.D., Sanders, S.P. \& Borow, K.M. Physiologic hypertrophy: effects on left ventricular systolic mechanics in athletes. J Am Coll Cardiol 1987, 9: 776-783.

7. Longhurst, J.C., Kelly, A.R., Gonyea, W.J. \& Mitchell, J.H. Chronic training with static and dynamic exercise: cardiovascular adaptation, and response to exercise. Circ Res 1981, 48 (Suppl.): 1-171-I-178.

8. Keul, J., Dickhuth, H.-H., Simon, G. \& Lehmann, M. Effect of static and dynamic exercise on heart volume, contractility, and left ventricular dimensions. Circ Res 1981. 48 (Suppl.): I-162-I-170. only mild clinical symptoms. In these cases, failure to recognize this underlying pathology may result in sudden and unexpected death. ${ }^{4,9}$ It is evident that endomyocardial biopsies are pertinent in the investigation of athletes who present with an enlarged heart but this procedure should only be undertaken if other investigations are unable to exclude pathology.

9. Maron, B.J., Roberts, W.C., McAllister, H.A., Rosing, D.R. \& Epstein, S.E. Sudden death in young athletes. Circulation 1980, 62: 218-229.

10. Henry, W.L., Clark, C.E. \& Epstein, S.E. Asymmetric septal hypertrophy (ASH): echocardiographic identification of the pathognomonic anatomic abnormality of IHSS. Circulation 1973, 47: 225-233.

11. Olsen, E.G.J. Morbid anatomy and histology in hypertrophic obstructive cardiomyopathy. In: Wolstenholme, G.E.W., O'Connor, M. (eds) Hypertrophic Obstructive Cardiomyopathy. Ciba Foundation Study Group No. 37, J. \& A. Churchill, London 1971, pp. 183-204.

12. Noorden, S. Van, Olsen, E.G.J. \& Pearce, A.G.E. Hypertrophic obstructive cardiomyopathy. A histological, histochemical and ultrastructural study of biopsy material. Cardiovasc Res 1971, 5: 118-131.

13. Archard, L.C., Bowles, N.E., Olsen, E.G.J. \& Richardson, P.J. Detection of persistent coxsackie B virus RNA in dilated cardiomyopathy and myocarditis. Eur Heart $J$ 1987, 8: 437-440. 